UCRL-JC-130635

PREPRINT

\title{
Probing Nano-defects in Fused Silica by Near-Field Scanning Optical Microscopy
}

\author{
Li Wang \\ M. Yan \\ W. Siekhaus \\ S. Oberhelman
}

This paper was prepared for submittal to

Journal of Applied Physics

March 17, 1998






\section{DISCLAIMER}

This document was prepared as an account of work sponsored by an agency of the United States Government. Neither the United States Government nor the University of California nor any of their employees, makes any warranty, express or implied, or assumes any legal liability or responsibility for the accuracy, completeness, or usefulness of any information, apparatus, product, or process

disclosed, or represents that its use would not infringe privately owned rights. Reference herein to any specific commercial product, process, or service by trade name, trademark, manufacturer, or otherwise, does not necessarily constitute or imply its endorsement, recommendation, or favoring by the United States Government or the University of California. The views and opinions of authors expressed herein do not necessarily state or reflect those of the United States Government or the University of California, and shall not be used for advertising or product endorsement purposes. 


\title{
Probing Nano-defects in Fused Silica by Near-Field Scanning Optical Microscopy
}

\author{
Li Wang, M. Yan, W. Siekhaus, and S. Oberhelman \\ Lawrence Livermore National Laboratory \\ Livermore, CA 94550
}

\begin{abstract}
Nano-defects were artificially introduced into fused silica surface by nano-indenting with a commercial atomic force microscope (AFM). The sample was illuminated at total internal reflection configuration and evanescent waves were detected by near-field scanning optical probe in the constant tip-sample separation mode. The strain fields associated with non-indents were attributed to the contrast mechanism in optical images. Thus the optical image directly maps out the strain distributions associated with these nano-indents. Optical images were taken at different polarizations of the incident light ( $s$ and $p$ ). Due to different field distributions near sample surface for the two polarizations, strain distributions at different depth can be probed. The spatial resolution of this technique is limited by the probe aperture size and detector sensitivity. This technique may be a useful tool to study laser-induced damage mechanisms in optical materials in microscopic scale.
\end{abstract}




\section{INTRODUCTION:}

Near-field optics overcomes the diffraction limit imposed by conventional optics and allows us to study optical properties of materials in sub-micron scale. Compared to atomic force microscopy (AFM), near field scanning optical microscopy (NSOM) has the main advantage of providing optical images in addition to topographic images. Although the spatial resolution of an AFM is much superior to that of a NSOM, optical information has proven to be more valuable to materials research on microscopic scale in many circumstances, especially in the case where material properties are not closely related to surface morphology. In this paper, we present experimental study of strain distributions associated with nano-indents in fused silica by using NSOM. The motivation behind this work lies in understanding of laser induced damage mechanisms in fused silica in high power optical systems. Previous work by several groups ${ }^{1,2}$ showed that several mechanisms may be responsible for laser induced damage in this material, such as local stress, polishing induced surface as well as sub-surface cracks, and various contaminants. However, no clear picture is available due to the lack of probing techniques of submicron resolution. Our goal is to develop such a technique that will enable us to study laserinduced damage mechanisms in fused silica on microscopic scale. In this technique, we illuminate the sample surface under study with a laser light at total internal reflection configuration. The evanescent wave is then collected by a scanning NSOM probe. By monitoring the intensity change of the collected signal, we can map out any perturbations in the material induced by stress and other defects. To demonstrate the feasibility of this technique, we did this experiment on a sample with intentionally introduced nano-indents. We hope to probe the stress fields associated with these indents at submicron resolution.

\section{EXPERIMENTAL:}

The nano-cracks were artificially introduced into a pristine fused silica sample surface through nano-indenting with a Dimension series AFM manufactured by Digital 
Instruments. The indenting tip is a three-sided diamond pyramid with apex angle of $60^{\circ}$.

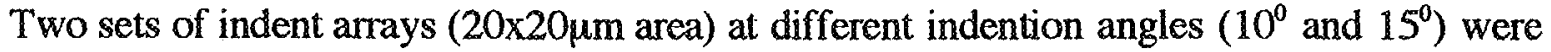
made. The angles were set by adjusting the control voltage of piezo-scanner that controls the indention tip. Each set consists of indents with different sizes. After indentation, the sample was scanned in situ with the same AFM. AFM images show that the size of these indents range from $80 \mathrm{~nm}$ to $100 \mathrm{~nm}$ in $x y$ plane and 1 to $4 \mathrm{~nm}$ in vertical $Z$ direction. The sample is then transferred to our homemade NSOM for imaging.

The schematic of the experimental setup is shown in figure 1. The sample was illuminated in the total internal reflection configuration (TIR) with 514nm line from an Ar ion laser. The angle of incidence was $65^{\circ}$, well above the critical angle of TIR, $41^{\circ}$. The focal point of the laser beam was aligned to the indented surface. A scanning fiber tip with an aperture size about $100 \mathrm{~nm}$ was used to collect the evanescent wave from sample surface in close proximity. The sample-tip separation was maintained constant $(15 \mathrm{~nm})$ by shear force feedback mechanism during scanning. The flat end of the fiber was input into a uncooled Hamamatsu photomultiplier sensor for signal detection. A half-wave plate polarizer was used to change the polarization of the incident light.

\section{THEORY:}

In the total internal reflection configuration, the amplitude of the evanescent field decays exponentially with vertical distance $Z$, normal to the TIR boundary, is given by $\mathrm{E}_{\text {vanescent }}=\mathrm{E}_{0} \exp \left(-z / \mathrm{d}_{\mathrm{p}}\right)$, where $\mathrm{E}_{0}$ is the amplitude of the field at the boundary associated with the photon in fused silica sample, $d_{p}$ is the penetration depth in air of $E_{p} \cdot d_{p}$ is given by ${ }^{3}$ $\mathrm{d}_{\mathrm{p}}=\lambda /\left[2 \pi\left(\sin ^{2} \theta-\eta_{21}{ }^{2}\right)^{1 / 2}\right]$,

where $\lambda$ is the wavelength in fused silica, $\theta$ is the angle of incidence, and $\eta_{21}$ is the index ratio between silica versus air. 
By introducing a fiber tip in close proximity to the TIR boundary, the tip will frustrate TIR due to its higher index as compared to air. Consequently, evanescent light will couple into fiber tip and become propagating light. The signal intensity detected at the other end of the fiber is given by

$S=C \cdot\left[E_{0} \exp \left(-z / d_{p}\right)\right]^{2}$,

where $C$ is the coupling coefficient of the fiber tip and depends strongly on the configuration of the tip. For a given tip, $\mathrm{C}$ can be treated as a constant.

During scanning, the tip to sample distance $(\mathrm{z})$ is kept constant. From above equations, any variation of the detected signal beyond noise level comes from the local index change in the sample. Based on this principle, we will be able to probe material properties in submicron scale.

The field distribution near TIR boundary within the sample can be worked out by solving classical electromagnetic equations with proper boundary conditions for different polarizations ${ }^{4,5}$. Standing wave pattern forms within the sample in TIR configuration. The relative field intensity distributions for $s$ and $p$ polarized light near sample surface are plotted in fig. 2 for incidence angle of $60^{\circ}$. In s polarization, the electric field is parallel to the sample surface $\left(\mathrm{E}_{\mathrm{s}}\right)$; while in $\mathrm{p}$ polarization, the electric field consists of components tangential to surface $\left(\mathrm{E}_{\mathrm{p} / t}\right)$ and perpendicular to surface $\left(\mathrm{E}_{\mathrm{p} \iota}\right)$. Fig. 2 shows the relationship between the standing wave intensity patterns for $s$ and $p$ tangential components. Except a factor of 4 higher intensity for $\mathrm{s}$, the two patterns are nearly $180^{\circ}$ out of phase. At the interface, the intensity for $s$ is about twice of that for $p$. However, $p$ tangential has a antinode located about $35 \mathrm{~nm}$ beneath the surface for the angle of incidence used in this experiment. Fig.2 also shows that the normal component of p polarization has a antinode near the surface with no significant light intensity. Even so, we will show that this component doesn't contribute to the optical signal detected by the fiber tip. The nanoindents are much smaller compared to the wavelength, so we can treat them as Raleigh 
scattering sites with large polarisability. The radiation field emitted by these dipole like sites excited by normal field component will have its maximum along the sample surface and no light along the surface normal. Therefore, it will not be collected by the fiber tip located normal to the surface. On the other hand, radiation excited by tangential field will have its maximum normal to the surface and be coupled into the fiber tip. Since $s$ and $p$ tangential field have different intensity distributions at different depths relative to the surface, optical images obtained at two polarizations will give us information on surface and subsurfaces. S polarization is more sensitive to changes at the surface; while $p$ polarization is more sensitive to features located right beneath the surface $(\sim 35 \mathrm{~nm})$. Other antinodes located more than $100 \mathrm{~nm}$ away from the surface will not contribute to detected signal because the near-field collection distance is less than $\lambda_{\text {silica }} / 2$ or $100 \mathrm{~nm}$ in our experiment.

\section{RESULTS AND DISCUSSION:}

Figs. $3 a$ and $3 b$ show the optical images at $s$ and $p$ polarizations, along with the corresponding topographs for the $10^{\circ}$ indents. Fig. $3 \mathrm{~b}$ is a higher resolution image with only one indent in it. Let us first examine the contrast mechanism for the optical images. Since the tip-sample separation was kept constant during scans, topographic features associated with these indents shouldn't contribute to the optical images $(z, \theta, C$ are fixed in eqn.1). However, possible optical artifacts caused by $Z$ motion crosstalk need to be examined carefully. According to Hecht et al. ${ }^{6}$, these artifacts occur because of the $z$ motion of the tip caused by tip-sample gap control, i.e.

$\delta S=\partial S /\left.\partial z\right|_{z} \cdot \delta z$

Because of the crosstalk, these artifacts show exact correlation with topographic structures and share the same resolution. For verification, these artifacts are negligible in our images because the optical images have very different resolutions compared to their corresponding topographs. Optical artifacts due to other parameters, such as feedback control electronics and scan direction, are also negligible for the same reason. 
Once we excluded all possible artifacts on the optical images, we confirmed that the nature of optical images is of pure dielectric contrast. We interpret the optical features associated with these nano-indents in term of strain distribution induced by nano-indenting. The exact strain fields surrounding the nano-indents are very complicated, and we will only present a qualitative picture based on experimental data. For fused silica, its refractive index decrease with tensile strain and increase with compressive strain ${ }^{7}$. Thus, we believe that the bright and dark features on the images correspond to areas of tensile and compressive stress around the indents. The fact that optical features are much bigger than their topographic counterparts is consistent with strain propagation around cracks. We notice that the optical features are not symmetric. Since the indenting tip we used was a three-side pyramid, naturally we would expect a symmetric strain map. However, the tip indented at a angle, so asymmetric stress distribution results. We also notice that the dark region exhibits arc shape rather than triangular shape. The mechanism behind it is not known, but we suspect that it might be also caused by the tilted indention. We have hoped to make indents at 0 degrees where the stress distribution is symmetric and less complicated; however, such a precision is difficult to achieve in nanometer scale.

By careful examination, we notice that there are optical features not associated with nano-indents in fig.3a. We attribute those features to other surface and sub-surface defect structures native to the sample. To demonstrate the sensitivity of this technique, a much smaller indent, barely seen on topograph (next to the middle indent), showed up nicely on optical images in fig.3a.

Let us now look at the polarization dependence of the optical images. Based on the theory we described earlier, the image obtained at s polarization reflects fcatures on the surface, whereas the image obtained at $p$ polarization reflects features right beneath the surface ( $35 \mathrm{~nm})$. Because of spatial dependence of stress distribution, we should see a difference for images taken at two polarizations. The difference between optical features for the two polarizations is indeed obvious in fig. 3 . The shape of the dark region changed from 
arc-like on the surface to rectangle-like away from the surface. Since strain alleviates during propagation, the transition from sharp feature to more smooth feature further justifies our interpretation. The sharper image in $s$ polarization as compared to that in $\mathrm{p}$ is a direct consequence of the much stronger electric field associated with s.

Next, we examine the dependence of optical image on the indention angle. Fig.4 is a schematic illustration of indentions at two tilting angles. The difference is exaggerated for clarity. Figs.5 (a) and (b) compare the images at $\mathrm{s}$ and p polarizations for indents made at two tilting angles. All images were taken under identical experimental parameters. Again we see a slight shifting of optical images relative to their corresponding topographs. Each indent in fig.5(b) has a much smaller indent on its right hand side and both of them show up clearly on optical images. At $15^{\circ}$ indention angle, the difference between images at two polarizations is also evident, with elongated dark features and very bright features on the surface and round like dark features and faint bright features away from the surface. If we compare images at same polarization between two indention angles, we observe striking differences, although there is only slight change in angle. We see changes in shape and relative position of bright and dark areas. Closer examination of topographic features in fig.5 shows that the features look almost identical in two cases. Even though indentions were made at different angles, the surface morphology doesn't change noticeably and this is the situation where optical images by NSOM comes to play. The change in indention angle induces change in the strain distribution around the indents, which is reflected in optical images.

Finally, the stress fields associated with nano-indents in these configurations are rather complicated and rigorous theoretical calculation is needed to provide a basic picture. The stress pictures presented herc are inevitably limited by the resolution and accurate interpretation of experimental data. Our intcrpretation of the data in terms of strain field distributions has to be further verified by other experiments such as NSOM based Raman and fluorescence spectroscopy that are currently in progress. 


\section{CONCLUSION:}

In conclusion, we have studied nano-indents on fused silica surface using NSOM in collection mode at total internal reflection configuration. We attribute the strain field distribution associated with nano-indents to the contrast mechanism for the optical images. Images obtained at different polarizations yield stress distributions at different depths relative to the surface. We showed that this technique is very sensitive in probing stress properties by indenting at slightly different tilting angles. We hope to use this technique to probe stress distributions in polished layers of fused silica where laser-induced damage often occurs in high power applications.

This work was performed under the auspices of the U.S. Department of Energy by Lawrence Livermore National Laboratory under contract No. W-7405-Eng-48. 


\section{References:}

1. A. A. Kozlov, A. A. Andreev, S. A. Chizhov, Proc. SPIE 3093, 75 (1997)

2. D. Du, X. Liu, G. Korn, J. Squier, IQEC'94, Opt. Soc. America, vol.9, p. 230 (1994)

3. J. M. Guerra, Appl. Optics, 29, 3741 (1990)

4. P. A. Temple, Appl. Optics, 20, 2656 (1981)

5. C. F. Kranenberg and K. C. Jungling, Appl. Optics, 33, 4248 (1994)

6. B. Hecht, H. Bielefeldt, Y. Inouye, and D. W. Pohl, J. Appl. Phys. 81, 2492 (1997)

7. M. Born and E. Wolf, Principle of Optics: Electromagnetic Theory of Propagation, Interference and Diffraction of Light (Pergamon, Oxford, 1993), p. 87-88. 


\section{Figure Captions:}

Figure 1: schematic diagram of experimental setup.

Figure 2: calculated standing wave intensities for $s$ and $\mathrm{p}$ polarizations near interface within the sample for incidence angle $\theta=60^{\circ}$.

Figure 3: optical images at $\mathrm{s}$ and $\mathrm{p}$ polarizations along with corresponding topographs for $10^{\circ}$ indents: (a) $4 \times 4 \mu \mathrm{m}$ images; (b) $0.5 \times 0.5 \mu \mathrm{m}$ high resolution images.

Figure 4: illustration of two types of indents at different tilting angles: $10^{\circ}$ and $15^{\circ}$.

Figure 5: comparison of optical images at $s$ and $p$ polarizations along with topographs for indents at different tilting angles: (a) $10^{\circ}$; (b) $15^{\circ}$. 




Fig. I 


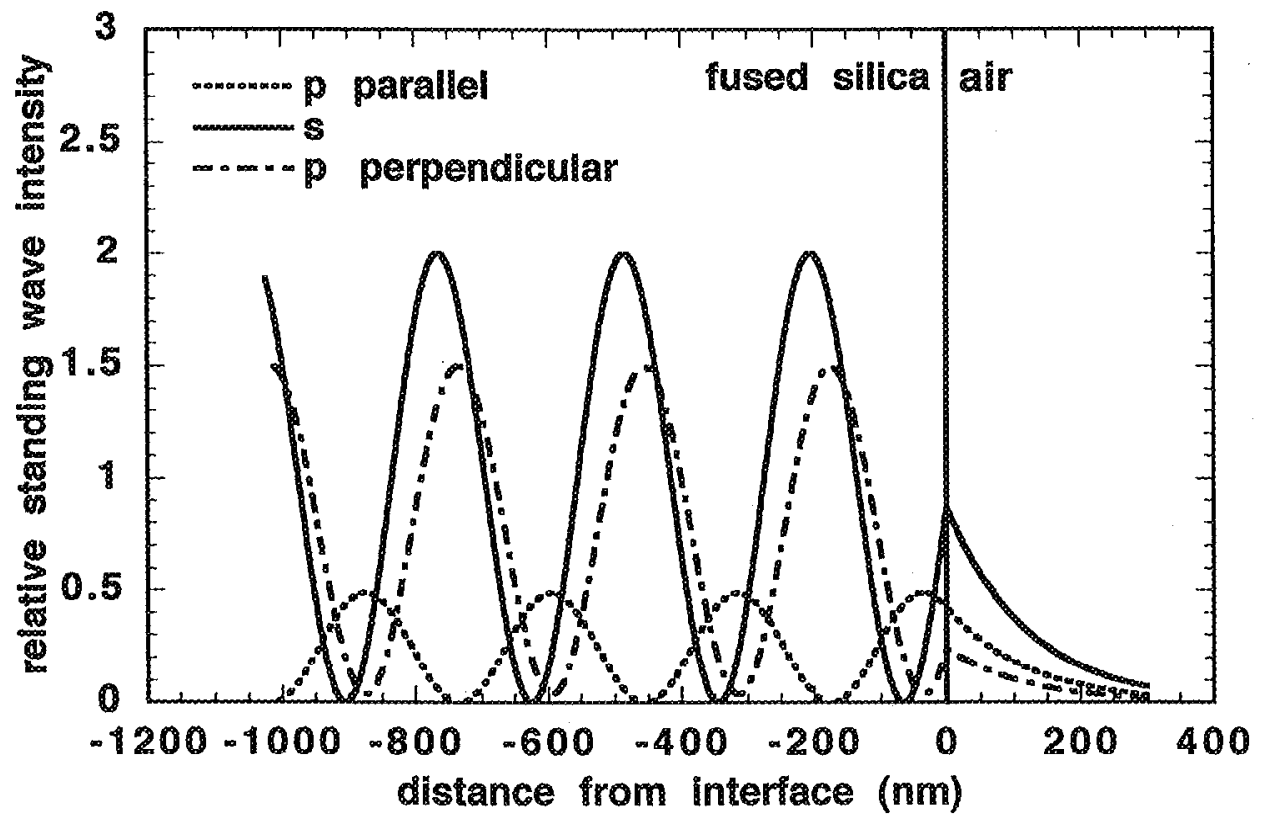

Fig. 2 


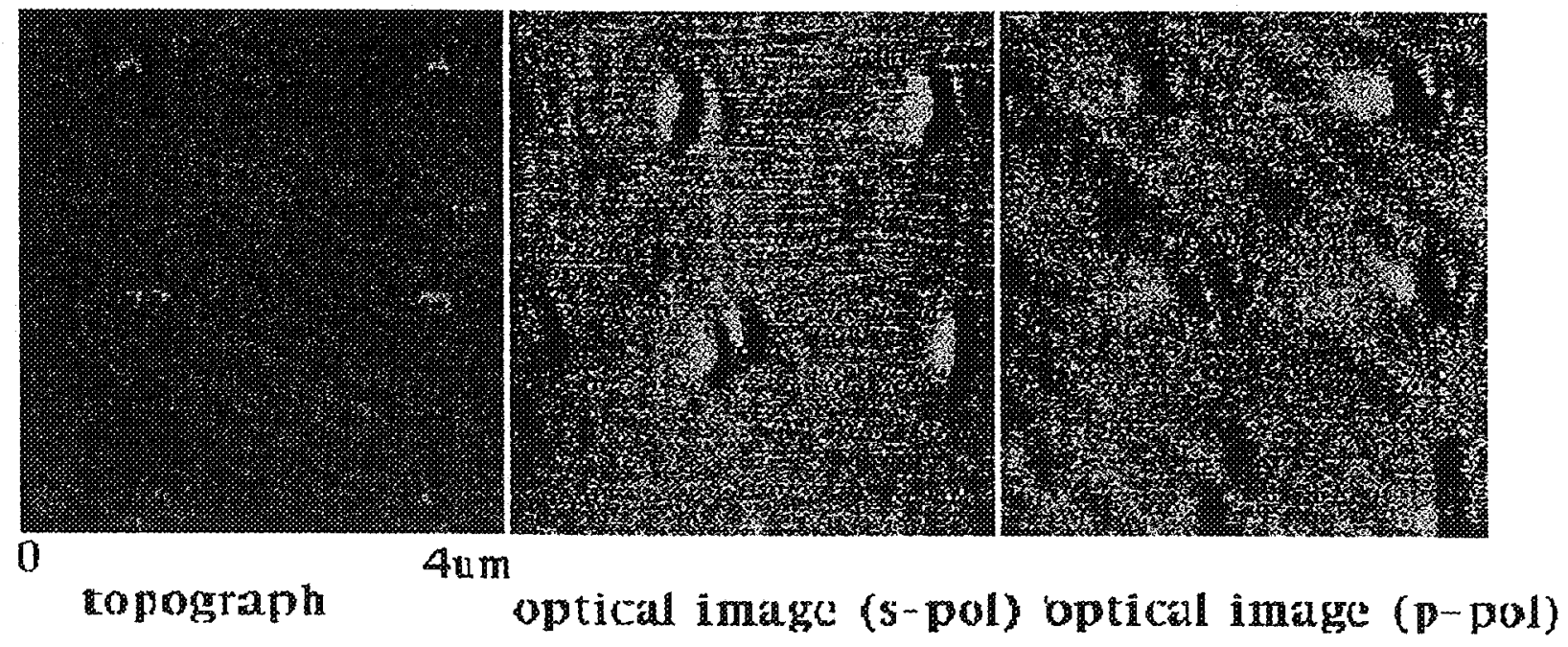

Fg. 3-a 


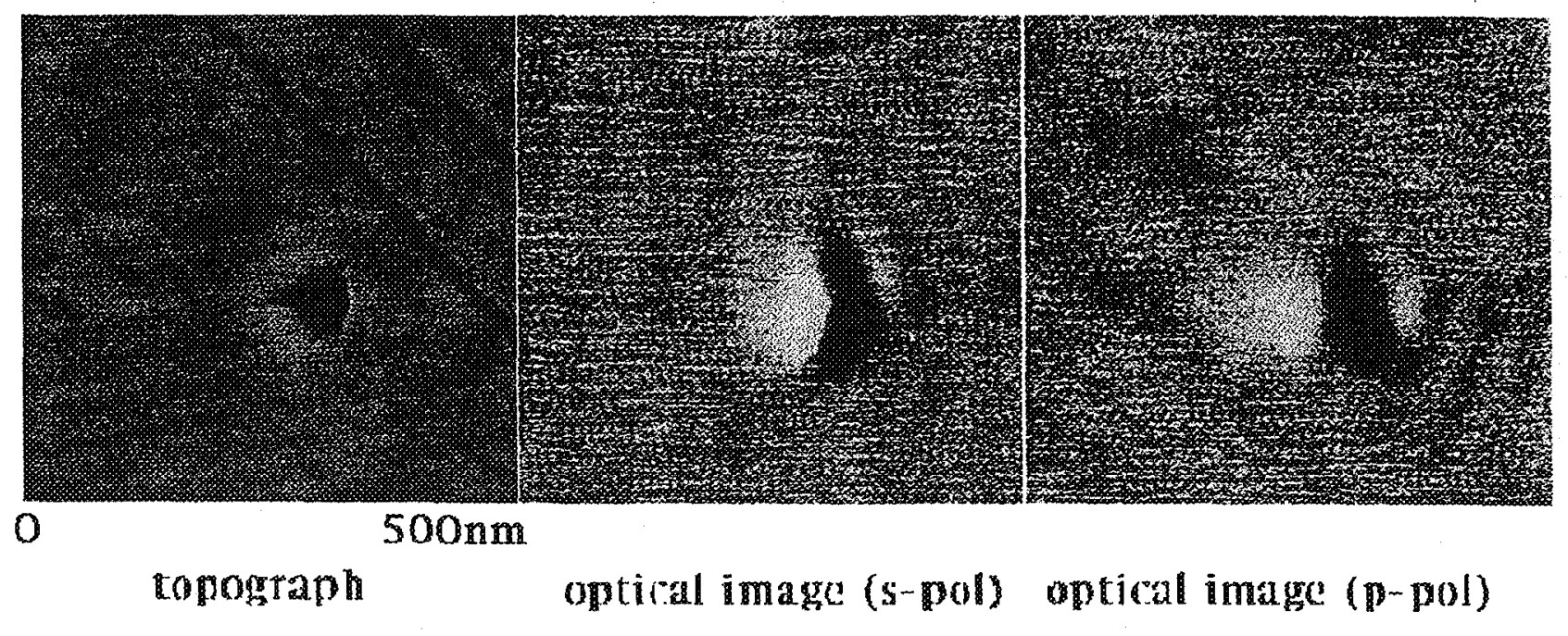

Fig $3-b$ 





(a)
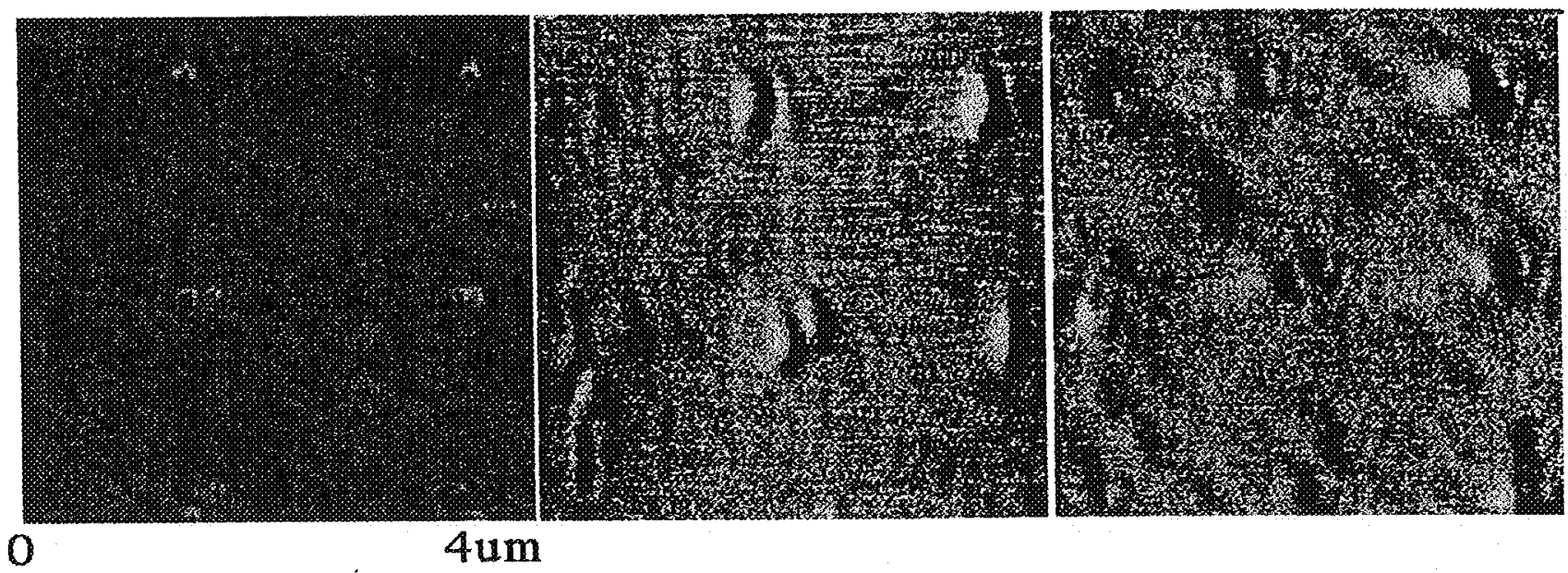

o

$4 u m$
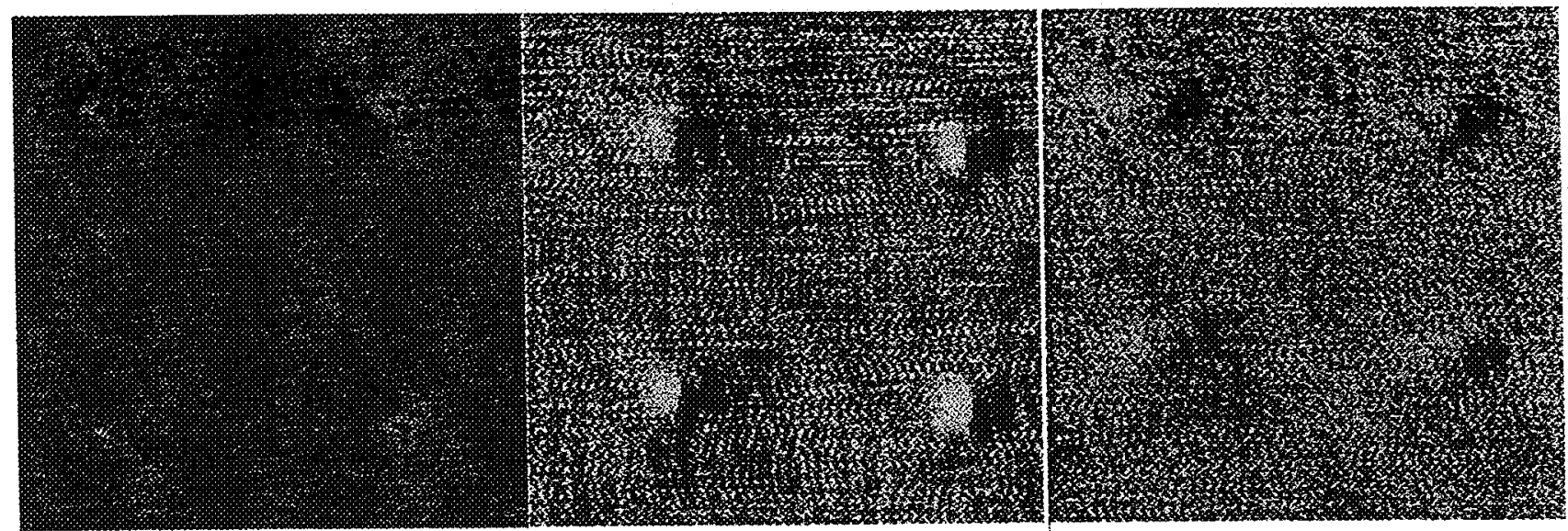

0

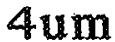

topograph

optical image (s-pol) optical image (p pol) 